\title{
Ileal Inflammation May Trigger the Development of GP2-Specific Pancreatic Autoantibodies in Patients with Crohn's Disease
}

\author{
Polychronis Pavlidis, ${ }^{1,2}$ Ourania Romanidou, ${ }^{1,3}$ Dirk Roggenbuck, ${ }^{4,5}$ \\ Maria G. Mytilinaiou, ${ }^{1,3}$ Faris Al-Sulttan, ${ }^{2}$ Christos Liaskos, ${ }^{3}$ Daniel S. Smyk, \\ Andreas L. Koutsoumpas, ${ }^{1,2,6}$ Eirini I. Rigopoulou, ${ }^{6}$ Karsten Conrad, ${ }^{7}$ \\ Alastair Forbes, ${ }^{2}$ and Dimitrios P. Bogdanos $1,3,6$ \\ ${ }^{1}$ Division of Transplantation Immunology and Mucosal Biology, King's College London School of Medicine at King's College Hospital, \\ London SE5 9RJ, UK \\ ${ }^{2}$ Department of Gastroenterology and Clinical Nutrition, University College Hospital, 250 Euston Road, London NW1 2PG, UK \\ ${ }^{3}$ Cellular Immunotherapy and Molecular Immunodiagnostics, Center for Research and Technology, Thessaly, 41222 Larissa, Greece \\ ${ }^{4}$ Faculty of Natural Science, Lausitz University of Applied Sciences, 01968 Senftenberg, Germany \\ ${ }^{5}$ GA Generic Assays GmbH, L.-Erhard-Ring 3, Dahlewitz, 15827 Berlin, Germany \\ ${ }^{6}$ Department of Medicine, University of Thessaly Medical School, Viopolis, Larissa 41110, Greece \\ ${ }^{7}$ Institute of Immunology, Technical University Dresden, Fetscherstrasse 74, 01307 Dresden, Germany \\ Correspondence should be addressed to Dimitrios P. Bogdanos, dimitrios.bogdanos@kcl.ac.uk
}

Received 22 June 2012; Accepted 7 September 2012

Academic Editor: Xavier Bossuyt

Copyright (๑) 2012 Polychronis Pavlidis et al. This is an open access article distributed under the Creative Commons Attribution License, which permits unrestricted use, distribution, and reproduction in any medium, provided the original work is properly cited.

\begin{abstract}
Why zymogen glycoprotein 2 (GP2), the Crohn's disease (CD)-specific pancreatic autoantigen, is the major target of humoral autoimmunity in inflammatory bowel diseases (IBD) is uknown. Recent evidence demonstrates that GP2 is also present on the apical surface of microfold (M) intestinal cells. As the colon lacks GP2-rich M cells, we assumed that patients with colonic CD are seronegative for anti-GP2. Anti-GP2 antibodies were tested in 225 CDs, including 45 patients with colonic location (L2), 45 with terminal ileum (L1) and 135 with ileocolonic involvement; 225 patients with ulcerative colitis (UC) were also tested. AntiGP2 reactivity was detected in $59(26.2 \%)$ CDs and $15(6.7 \%)$ UCs $(P<0.001)$. Only 5 CDs with L2 had anti-GP2 antibodies, compared to $54 / 180(30.0 \%, P=0.0128)$ of the CDs with L1 and L3. Anti-GP2 antibody positive CD patients had higher ASCA titres compared to seronegative cases. Amongst the $128 \mathrm{CD}$ patients with previous surgical intervention, $45(35.0 \%)$ were anti-GP2 antibody positive compared to $14 / 97(14.0 \%)$ without surgical $(P<0.001)$. Our data support the assumption that ileal inflammation is required for the development of anti-GP2 antibodies in $\mathrm{CD}$, and suggest that the intestine rather than the pancreatic juice is the antigenic source required for the initiation of anti-GP2 antibodies.
\end{abstract}

\section{Introduction}

Pancreatic autoantibodies $(\mathrm{PAB})$ detected by indirect immunofluorescence (IIF) are specific markers of Crohn's disease (CD), being present in approximately $27-39 \%$ of patients with this condition, but in fewer than $8 \%$ of patients with ulcerative colitis (UC) or other disorders unrelated to inflammatory bowel diseases (IBD) [1-7]. The major target antigen of $\mathrm{PAB}$ has recently been elucidated as a pancreatic glycosyl phosphoinositol (GPI) membraneanchored protein, also known as zymogen glycoprotein 2 (GP2) [8].

It was previously believed that GP2 was exclusively expressed by pancreatic acinar cells $[9,10]$, but recent studies have clearly demonstrated that GP2 is also located in the microfold (M) cells of the follicle-associated epithelium (FAE) of intestinal Peyer's patches [11]. Thus, it appears that GP2 is located in the intestine, as well as the exocrine 
pancreas, and this may explain its intriguing autoantigenicity in patients with CD [9-13].

Direct proof of the relationship between the autoantigenicity of GP2 and its peculiar location on the apical surface of the GP2-rich intestinal M cells has not yet been obtained [12]. PCR analysis of colonic biopsy material of anti-GP2 antibody positive patients with CD suggested that there is a CD-specific overexpression of GP2 in this disease [8], but the data are scarce and far from conclusive [12].

While $M$ cells are found in abundance in the small intestine and in particular in the ileum, they are hardly detectable in the large intestine [14]. We assumed that the production of GP2 autoantibodies is triggered during ileal inflammation and that high expression of GP2 by M cells in the inflamed ileal environment is important for the release of this antigen and its continual exposure to the immune system [12]. If this holds true, it would be expected that patients with exclusively colonic CD would lack anti-GP2 antibodies as compared to patients with ileal or ileocolonic inflammation. Such information would also provide clues as to whether GP2 autoantibodies participate in the immunopathogenicity of CD or are just epiphenomena, secondary to ileal inflammation.

\section{Patients and Methods}

2.1. Patients. Serum samples of 450 patients from a cohort of 854 follow-up IBD patients seen in the outpatient clinics of one of the authors (A. Forbes) who runs a tertiary referral service in the UK (currently at University College Hospital, London) were tested.

The study population included 225 patients with CD (men/women: 98/127, $36.0 \pm 14.3$ years; disease duration $13.0 \pm 10.1$ years) and $225 \mathrm{UC}$ patients (male/female: 113/112; age median: $51.0 \pm 15.7$; disease duration median: $14.0 \pm 12.9$, Table 1$)$.

The diagnoses of CD and UC were based on current standard clinical, radiological, endoscopic, and histological criteria (Lennard-Jones criteria) [15]. The disease phenotype was determined based on the Montreal classification [16].

Disease location was the criterion for the selection of CD patients. All the patients with ileal $(\mathrm{L} 1=45)$ and colonic (L2 = 45) involvement were included. A proportionally larger group of patients with extensive disease (ileal and colonic involvement, L3 = 135) was selected reflecting the higher prevalence in the original population. An equal number of patients with UC were randomly selected.

Follow-up samples were taken from 40 opportunistically selected patients (CD:20, UC:20) at various time points (median CD follow up of $3.0 \pm 1.3$ years; median UC follow up: $3.0 \pm 1.0$ years).

In addition, 75 serum samples from 50 healthy blood donors and 25 patients with irritable bowel syndrome have been included as normal and pathological controls, respectively. Laboratory, histological and clinical data recorded in an electronic database were used to analyse patients stratified into groups according to the presence or not of anti-GP2 antibodies.
The study was conducted in accordance with the Helsinki declaration and approved by the local ethics committees. Written informed consent was obtained from each individual. All sera had been stored at $-20^{\circ} \mathrm{C}$ before analysis.

2.1.1. Detection of Anti-GP2 Antibodies by ELISA. IgG antiGP2 antibodies were tested in serum samples of patients with IBD by a commercial ELISA (Generic Assays, Dahlewitz/Berlin, Germany) [17], according to the manufacturer's instructions. The assay is based on recombinant human GP2 expressed in Spodoptera frugiperda 9 cells as solid-phase antigen, as described previously [18]. Briefly, the plasmid pcDNA3.1 + GP2-trunc-Thrombin-His was used which codes the amino acid sequence of GP2 isoform BAA88166 (pancreatic GP2 alpha form) corresponding to the formal isoform 2 (NP_001493) missing the last 8 amino acids at the N-terminal end [19]. The cutoff for positivity was set to $20 \mathrm{AU} / \mathrm{mL}$, as recommended by the manufacturer. The anti-GP2 IgG ELISA displayed an intra-assay variability of $4.3 \%$ and an interassay variability of $5.6 \%$ for samples giving $29 \mathrm{AU} / \mathrm{mL}$ and $27 \mathrm{AU} / \mathrm{mL}$, respectively.

2.1.2. Detection of Antibodies to Saccharomyces cerevisiae (ASCA) by ELISA. In view of the high specificity of ASCA antibodies for $\mathrm{CD}$ and the frequent cooccurrence with pancreatic autoantibodies described in previous studies [8, 20], patients' serum samples were also tested for ASCA antibody reactivity. A commercially available ELISA (INOVA Diagnostics) kindly provided by Dr. Gary L. Norman was used for the quantitative determination of IgA and IgG ASCA antibodies, following the manufacturer's protocol. A cutoff for positivity was set to $25 \mathrm{AU} / \mathrm{mL}$, as recommended by the manufacturer. The intra-assay coefficient of variation was $3.7 \%$ for a sample containing $65 \mathrm{AU} / \mathrm{mL}$ of ASCA IgA and $4.5 \%$ for a sample containing $45 \mathrm{AU} / \mathrm{mL}$ of ASCA IgG. The inter-assay coefficient of variation was $4.5 \%$ for a sample containing $52 \mathrm{AU} / \mathrm{mL}$ and $2.5 \%$ for a sample containing $52 \mathrm{AU} / \mathrm{mL}$ of ASCA IgA and ASCA IgG, respectively.

2.2. Statistics. All statistical tests were performed using the SPSS 15.0 statistical software package (SPSS Inc., Chicago, Illinois, USA). Prizm software (by GraphPad Software Inc., La Jolla, California, USA) was used for drawing the presented figures. An assumption of nonparametric variables was made and the comparisons were performed with MannWhitney, Fisher exact, and chi-square tests as appropriate. Wherever required a non-parametric Spearman correlation was performed. Results are presented as percentages and medians with standard deviation error and odd ratios with 95\% confidence intervals (CI). All $P$ values reported are for two-tailed analysis.

\section{Results}

3.1. IgG Anti-GP2 Antibodies in CD, UC, and Non-IBD Controls. IgG anti-GP2 reactivity was detected in $59(26.2 \%)$ patients with CD and $15(6.7 \%)$ patients with $\mathrm{UC}\left(\chi^{2}=31.3\right.$, df $=1, P<0.000$, odds ratio: $4.98,95 \%$ CI 2.73 to 9.08$)$. 
TABle 1: Main demographic and clinical characteristics of the 225 patients with Crohn's disease (CD) and the 225 patients with ulcerative colitis (UC) included in the present study.

\begin{tabular}{|c|c|c|}
\hline & $\mathrm{CD}$ & UC \\
\hline$N$ & 225 & 225 \\
\hline $\operatorname{Sex}(m / f)$ & $98(43.6 \%) / 127(56.4 \%)$ & $113(50.2 \%) / 112(49.8 \%)$ \\
\hline Age $($ mean $\pm \mathrm{SD})$ & $36 \pm 14.3$ & $51 \pm 15.7$ \\
\hline Age at diagnosis (mean $\pm \mathrm{SD}$ ) & $23 \pm 11.6$ & $30 \pm 14.6$ \\
\hline Disease duration (mean $\pm S D$ ) & $13 \pm 10.1$ & $14 \pm 12.9$ \\
\hline \multirow{3}{*}{ Location $n(\%)$} & L1: $45(20 \%)$ & E1: $28(12.4 \%)$ \\
\hline & L2: $45(20 \%)$ & E2: $66(29.3 \%)$ \\
\hline & L3: $135(60 \%)$ & E3: $131(58.2 \%)$ \\
\hline \multirow{4}{*}{ Behaviour $n(\%)$} & B1: $106(47 \%)$ & \\
\hline & B2: $62(28 \%)$ & \\
\hline & B3: $57(25 \%)$ & \\
\hline & Perianal: 60 (27\%) & \\
\hline \multirow{3}{*}{ Age } & A1: $46(20 \%)$ & \\
\hline & A2: $156(70 \%)$ & \\
\hline & A3: $23(10 \%)$ & \\
\hline
\end{tabular}

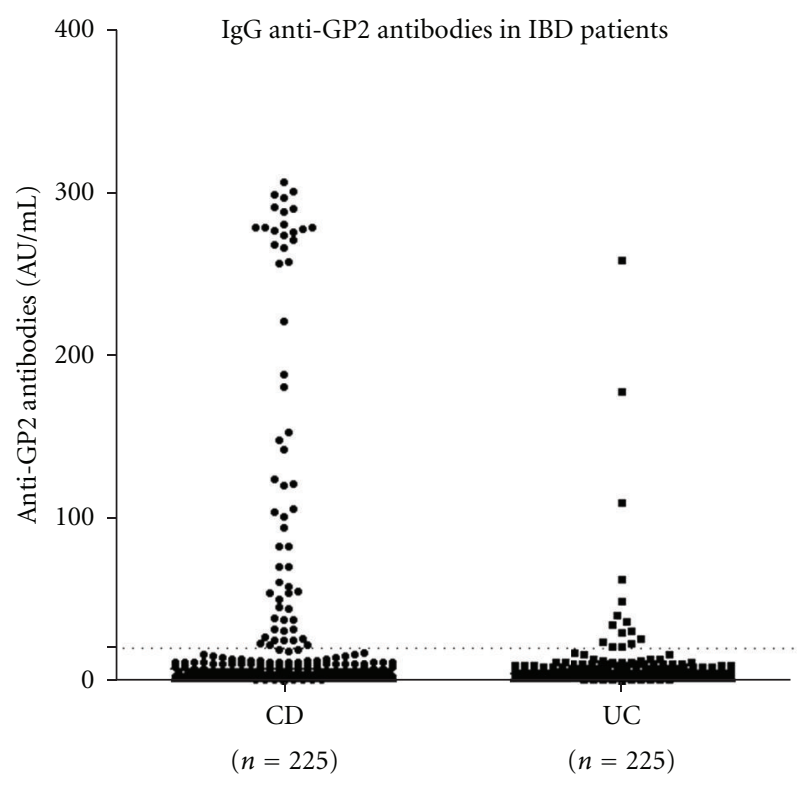

Figure 1: IgG anti-GP2 antibodies in 225 patients with Crohn's disease (CD) and 225 patients with ulcerative colitis (UC). A cutoff of $20 \mathrm{AU} / \mathrm{mL}$ established by the manufacturer of the commercial ELISA (Generic Assays) is indicated with a dot line.

The titres were significantly higher in $\mathrm{CD}$ in comparison to UC patients ( $U: 18920, P<0.0001$, Figure 1 ).

IgG antibodies were present in one $(1 / 75,1.33 \%$; $47 \mathrm{AU} / \mathrm{mL}$ ) non-IBD control tested (a 37-year-old female suffering from irritable bowel syndrome without family history of IBD).

The sensitivity of IgG anti-GP2 antibodies for IBD versus non-IBD controls was $16 \%$, the specificity $99 \%$, and the likehood ratio 12.33 . When comparing $\mathrm{CD}$ versus $\mathrm{UC}$ then the sensitivity was $26 \%$, the specificity $93 \%$, and the likehood ratio 3.93 .

3.2. IgG Anti-GP2 Antibodies in CD Patients according to Disease Location. CD patients with ileal (L1) or extensive disease (L3) presented higher prevalence of anti-GP2 IgG $(P=0.0128)$ with significantly higher titres as shown in Figure 2. Thus, anti-GP2 antibodies were present in 5/45 (11.1\%) L2 CD, representing just $8.5 \%(5 / 59)$ of the total anti-GP2 positive CD cohort and 2.2\% (5/225) of the total $\mathrm{CD}$ population included in the present study. This was statistically less prevalent compared to the 30\% (54/180) anti-GP2 seropositivity seen in patients with L1 and L3, who represent the $91.5 \%$ of the total anti-GP2 seropositive CD patients and $24 \%(54 / 225)$ of the total CD population.

3.3. IgG Anti-GP2 Antibodies versus ASCA (IgA and IgG). A summary of the results is given as a Venn diagram in Figure 3. Amongst the 225 patients with CD, 141 (62.7\%) and 99 (44.0\%) had IgG and IgA ASCA, respectively. Overall, 153 (68.0\%) CD patients had IgG and/or IgA ASCA compared to $28(12.4 \%)$ UC patients $(P<0.0001)$. Among the ASCA (IgA and/or IgG) positive CD and UC patients, $50(33 \%)$ and $5(18 \%)$ were positive for IgG anti-GP2, respectively. Overall, $57(40 \%)$ of the IgG and $38(38 \%)$ of the IgA ASCA positive patients had anti-GP2 antibody reactivity, respectively. Only 35 (15.6\% of the total 225) CD patients had simultaneous reactivity for ASCA (both IgG and IgA) and anti-GP2. These represented $59.3 \%$ of the total $(n=59)$ anti-GP2 antibody reactive cases. Among the 62 (28.0\%) ASCA seronegative CD patients, $9(15.0 \%)$ were positive for anti-GP2 IgG.

Although, there was no correlation between ASCA (IgA or IgG) and anti-GP2 titres in double positive patients, the titres of ASCA (IgA and IgG) were higher in patients positive for IgG anti-GP2 as shown in Figure 4. 


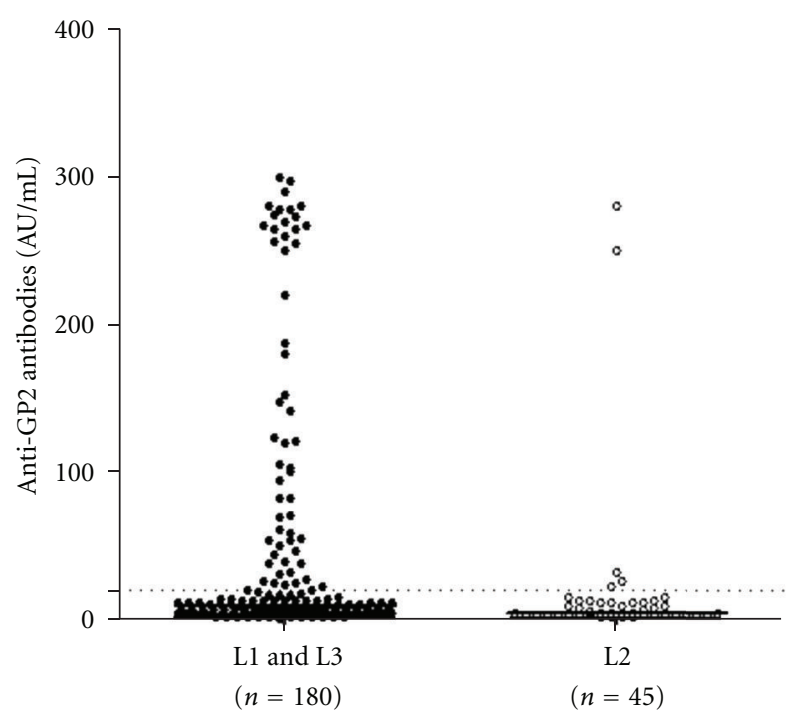

Figure 2: Anti-GP2 antibody titres in 225 patients with Crohn's disease (CD) stratified in two groups: patients with restricted colonic location (L2) and patients with ileal (ileal or ileocolonic) location (L1 and L3), according to Montreal classification.

There was no statistically significant difference in the prevalence of ASCA IgA in patients with different locations (L1+L3: 83/180 versus L2: $18 / 45, P=0.5056)$. Patients with colonic disease (L2) though had lower prevalence of ASCA IgG (L1+L3: 123/180 versus L2: 19/45, $P<0.0009$ ).

No difference was found in the prevalence of ASCA IgA in patients with different behaviour phenotypes (B1: 42/108, B2: 33/62 and B3: 24/57) but ASCA IgG were more prevalent in patients with stricturing disease (B1:58/108, B2:48/62, and $\mathrm{B} 3$ : $35 / 57 ; P=0.0131)$.

3.4. IgG Anti-GP2 Antibody Association with Other Clinical Parameters. There was no statistically significant difference between anti-GP2 IgG positive and negative CD patients in regards to age of disease onset and duration of disease (Figure 4). There was no correlation between anti-GP2 IgG titres and disease duration. The prevalence of IgG antiGP2 antibodies differed when CD patients were stratified in subgroups according to their disease behaviour (Figure 5), but there was no difference in the titre medians between subgroups. IgG anti-GP2 antibodies were present in 29/106 (27\%) CD patients with B1, 22/62 (35\%) with B2, and 8/57 (14\%) with B3 phenotype $(P=0.0046)$.

Family history of IBD was not associated with anti-GP2 antibody seropositivity. Among the 57 (25.3\%) CD patients with family history of IBD, $13(22.8 \%)$ were positive for antiGP2 IgG compared to 43 out of 168 (25.6\%) without family history of $\operatorname{IBD}(P>0.05)$.

Amongst the $128 \mathrm{CD}$ patients with previous surgical intervention, $45(35.2 \%)$ were positive for anti-GP2 IgG compared to only $14 / 97$ (14.4\%) CD patients without surgical history $\left(\chi^{2}=12.25, \mathrm{df}=1, P<0.000\right.$, odds ratio: $3.214,95 \%$ CI 1.641 to 6.298$)$.
The prevalence of anti-GP2 antibodies did not differ amongst naive patients or patients at early stages of their disease (duration of disease less than two years) compared to patients with $>2$ years disease duration $(4 / 22,18 \%$ versus $54 / 203,26 \%, P>0.05)$. Also, the prevalence of anti-GP2 antibody reactivity did not differ amongst patients treated with or without infliximab (11/38 and $28.9 \%$ versus $48 / 187$ and $25.7 \% ; P>0.05)$.

3.5. Behaviour of Anti-GP2 Antibodies over Time. An additional set of experiments was carried out to test the behaviour of anti-GP2 antibodies over time in 20 CD patients, including $8(20 \%)$ who were anti-GP2 antibody positive at baseline. All 8 anti-GP2 antibody positive patients have shown a decline of their GP2 autoantibody titres at repeated testing. Of those, 5 retained their seropositivity and 3 became seronegative. Of the 12 anti-GP2 antibody negative cases at baseline, 2 became seropositive at relatively low titres during follow up (Figure 6). The single UC patient who was positive on initial sampling remained positive on the second test.

\section{Discussion}

Pancreatic antibodies directed against GP2 have been considered serological markers of CD, being present in approximately $20-36 \%$ of patients with IBD [12, 17-19, 21-23]. Why GP2 becomes an autoantigenic target in CD is unclear [12, $13,24]$. Also, why some but not all patients with CD develop humoral autoreactivity against this pancreatic autoantigen remains elusive [12]. Moreover, it is not clear whether these autoantibodies are secondary to intestinal destruction or participate in the induction of the disease.

In the present study, we assumed that patients showing a disease location restricted to the colon, and therefore without inflammation of the ileum, would lack antibody reactivity to GP2 [12]. We based our hypothesis on recent evidence indicating that there is GP2 expression in the intestine in addition to its former known pancreatic site of synthesis. It seems to be limited to the intestinal $M$ cells [11]the atypical epithelial cells that account for up to $10 \%$ of FAE $[14,25,26]$. The role of $\mathrm{M}$ cells is generally to phagocytose macromolecules and microbes and to transport them to the underlying mucosa-associated immune system for antigen presentation. Thus, $\mathrm{M}$ cells play a crucial role in maintaining the critical balance in terms of recognising and differentiating self and nonself. Intriguingly, $\mathrm{M}$ cells and Peyer's patches are particularly abundant in the distal part of the ileum $[11,27]$, which has been considered to be the most likely site of inflammation onset in newly diagnosed, adolescent patients with CD and is generally one of the most common sites for clinically apparent disease activity [28]. According to the above argument, intestinal inflammation sparing the ileum would not be able to release GP2 from the inflamed tissue. The release of GP2 would be a prerequisite for the activation of the immune system and the initiation of an autoimmune reaction that could lead to the induction of anti-GP2 antibodies [12]. 
$\mathrm{CD}(n=225)$ pos. anti-GP2 $>20$, ASCA $>25 \mathrm{AU} / \mathrm{mL}$

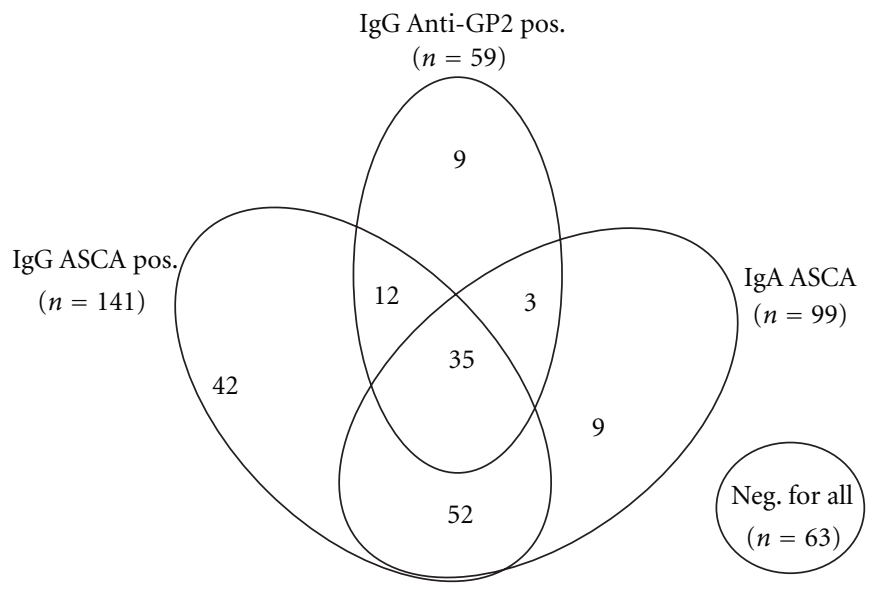

Figure 3: Venn Diagram showing IgA ASCA, IgG ASCA, and IgG anti-GP2 antibody reactivity of the 225 Crohn's disease (CD) patients.

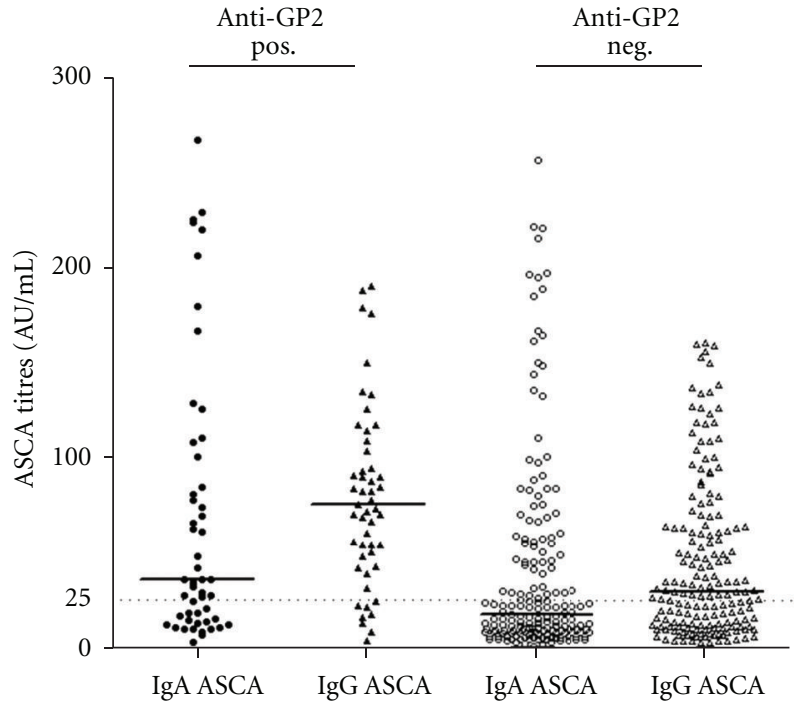

Figure 4: Comparison of IgA and IgG ASCA titres in IgG anti-GP2 antibody positive and negative patients with Crohn's disease (CD). Statistical analysis did not reveal significant differences amongst anti-GP2 antibody positive and anti-GP2 antibody negative CD patients in ASCA titres.

Indeed, our study clearly demonstrates that patients with the restricted colonic form of the disease do not show significant antibody reactivity against GP2 compared to those with a disease location that involves the ileum, the site of GP2-rich M cells. The necessity for ileal inflammation in order for anti-GP2 antibodies to be developed can also explain why only a minority of patients with UC have shown autoantibody reactivity against this antigen [12, 17-19, 2123]. In our study, fewer than $7 \%$ of 225 patients with UC have shown anti-GP2 antibodies. This percentage is in agreement with most studies reporting on the prevalence of anti-GP2 antibodies in smaller cohorts of UC patients [8, 17-19].
Anti-GP2 antibodies in CD patients according to disease behaviour

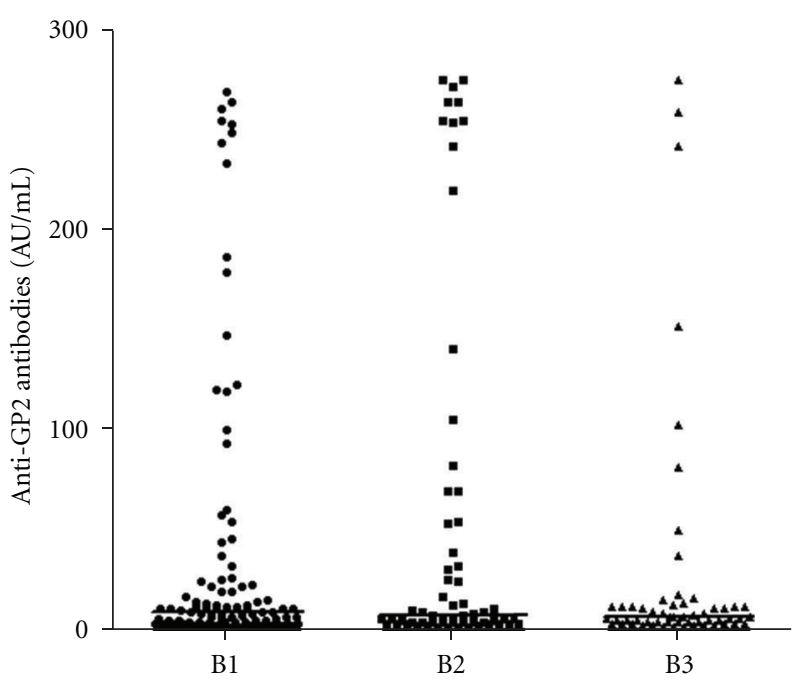

FIGURE 5: Comparison of IgG anti-GP2 antibodies in patients with Crohn's disease (CD) stratified in accordance to disease behaviour (Montreal Classification, B1, B2, and B3).

A previous study reporting coexistence of ASCA and anti-GP2 in a significant proportion of CD patients [17] has been followed by other studies that were unable to replicate this finding $[21,22]$. Also, comparison of various demographic and clinical parameters analysed in our cohorts was unable to show significant differences in terms of age of disease onset, as well as disease duration, in accordance with previous studies [21]. A lower prevalence was found in patients with penetrating disease B3 and this needs further investigation. Anti-GP2 antibodies were more prevalent in patients with previous surgical intervention than in those without (35\% versus $14 \%, P<0.001)$. The clinical relevance of this finding will remain uncertain until it is replicated in larger studies. Most studies conducted so far have been 


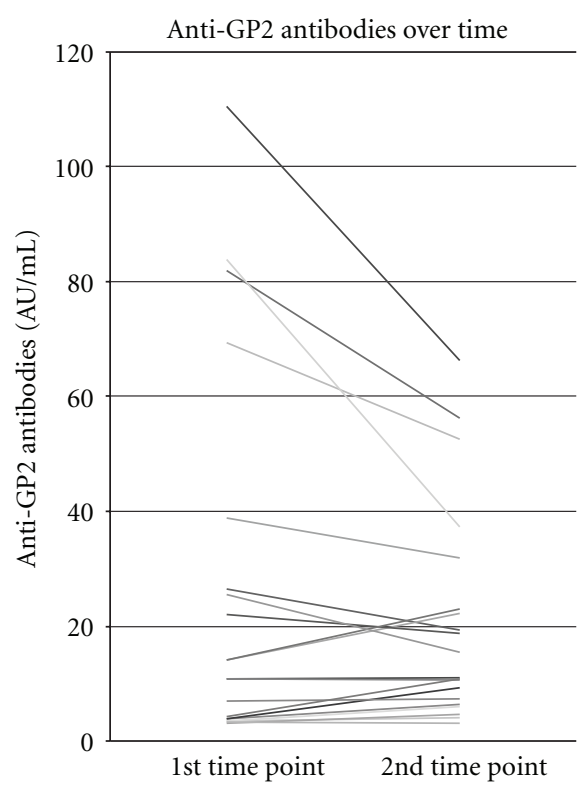

Figure 6: Behaviour of anti-GP2 antibodies during follow up in 20 randomly selected patients with Crohn's disease (CD), including 8 anti-GP2 antibody positive at baseline.

unable to provide a decisive outcome, and thus our data need to be interpreted with caution $[8,12,17,18,21-23]$.

Our study cannot estimate accurately the prevalence of anti-GP2 in patients with $\mathrm{CD}$, and this needs to be noted. Our cohort has an overrepresentation of patients with ileal involvement. Also, most serum samples originate from patients already treated. For an accurate estimation of the prevalence of anti-GP2 antibodies, a large cohort of naïve patients with Crohn's disease has to be tested. Taking into account that over the course of the disease, anti-GP2 antibodies decline, it would be logical to assume that the real prevalence of anti-GP2 antibodies may be higher than that so far reported in cohorts involving sera from already treated patients. Safer conclusions can only be reached if serial, large number of samples collected prospectively over a long-duration of follow up could be tested.

A few other points need to be made. The first point considers whether anti-GP2 antibodies contribute to the development of the disease or whether their existence is just an epiphenomenon following intestinal epithelial destruction [12]. Furthermore, it has been demonstrated that GP2 recognises FimH, which is a constituent of the type 1 pilus expressed on the outer membrane of some enterobacilli, such as E. coli and Salmonella enterica [11]. FimH have a lectinlike capability to bind certain glycoproteins in a mannosedependent manner, and appear to be able to recognise GP2 $[11,13]$. Specific interaction with FimH positive bacteria found in some but not all $\mathrm{CD}$ patients might explain the limited prevalence of anti-GP2 antibodies in IBD patients. Work on IBD animal models and GP2-deficient mice may shed a light on the pathogenic potential of GP2-specific immune responses. The fact that GP2 is expressed on the apical surface of $M$ cells $[11,13]$ making it accessible to antibodies is of special interest, as it would support the notion that these cells may be the targets of antibodydependent cytotoxicity [12]. On the other hand, a high proportion of individuals with $\mathrm{CD}$ appear seronegative for anti-GP2 antibodies, not only at baseline but also over time. This finding clearly indicates that the loss of immunological tolerance to this antigen is not an a priori condition for the development of the disease. There is no doubt that the pathogenesis of CD involves mechanisms other than those responsible for the induction of GP2 autoimmunity [29]. These mechanisms may be important for the establishment of the disease, acting in isolation or in combination with those leading to $\mathrm{M}$ cell-related induction of anti-GP2 antibodies, seen in over a quarter of patients with Crohn's disease. Why only those and not all patients develop these autoantibodies remains unclear. As anti-GP2 is absent in approximately $74 \%$ of patients with $\mathrm{CD}$, the validity of the isolated detection of GP2-specific PAB is impaired. Most investigators agree that the routine use of isolated serological markers for diagnosis and especially for the follow up of patients with inflammatory diseases is limited by their inadequate performance in terms of the diagnosis and prognosis of CD [29-31]. ASCA, for example, are more prevalent in patients with $\mathrm{CD}$ and their participation in the routine testing of patients with $\mathrm{CD}$ is more than adequate. In conclusion, we share with others the notion that several serological markers must be used in combination to be more effective compared to isolated/single marker testing.

Nevertheless, anti-GP2 antibody testing appears to be one of those tests that can be added in the diagnostic workup of patients with $\mathrm{CD}$. More work needs to be done over the next few years to understand the immunobiological role of this antigen and its relevance to IBD.

Emerging data indicating an important immunoregulatory role of GP2 for the emergence of innate and adaptive immunity (including the recruitment of regulatory $\mathrm{T}$ cells) in the intestine [32] may initiate an intense research in this field and elucidate the role of this interesting autoantigen.

\section{Conflict of Interests}

D. Roggenbuck is a shareholder of GA Generic Assays GmbH and Medipan $\mathrm{GmbH}$. The remaining authors declare that they have no competing financial interests. A. Forbes and D. P. Bogdanos equally contributed to this work.

\section{Acknowledgments}

$\mathrm{UCH}$ receives financial support for its research from the UK Clinical Research Service, National Institute for Health Research. D. P. Bogdanos was supported by the Higher Education Funding Council for England. O. Romanidou, D. Smyk and M. Mytilinaiou were supported by the European Association for the Study of Liver (EASL). The authors thank Dr. Gary L. Norman (INOVA Diagnostics) for providing the ASCA ELISA kits. Part of this work was presented as a poster at the 8th International Meeting on Autoimmunity, Granada, Spain (May 2012). 


\section{References}

[1] W. Stöcker, M. Otte, S. Ulrich et al., "Autoimmunity to pancreatic juice in Crohn's disease. Results of an autoantibody screening in patients with chronic inflammatory bowel disease," Scandinavian Journal of Gastroenterology, Supplement, vol. 139, pp. 41-52, 1987.

[2] W. Stöcker, M. Otte, S. Ulrich, D. Normann, K. Stöcker, and G. Jantschek, "Autoantibodies against the exocrine pancreas and against intestinal goblet cells in the diagnosis of Crohn's disease and ulcerative colitis," Deutsche Medizinische Wochenschrift, vol. 109, pp. 1963-1969, 1984.

[3] S. Desplat-Jégo, C. Johanet, A. Escande et al., "Update in AntiSaccharomyces cerevisiae antibodies, anti-nuclear associated anti-neutrophil antibodies and antibodies to exocrine pancreas detected by indirect immunofluorescence as biomarkers in chronic inflammatory bowel diseases: results of a multicenter study," World Journal of Gastroenterology, vol. 13, no. 16, pp. 2312-2318, 2007.

[4] F. Seibold, P. Weber, H. Jenss, and K. H. Wiedmann, "Antibodies to a trypsin sensitive pancreatic antigen in chronic inflammatory bowel disease: specific markers for a subgroup of patients with Crohn's disease," Gut, vol. 32, no. 10, pp. 1192-1197, 1991.

[5] F. H. Klebl, F. Bataille, C. Huy, F. Hofstädter, J. Schölmerich, and G. Rogler, "Association of antibodies to exocrine pancreas with subtypes of Crohn's disease," European Journal of Gastroenterology and Hepatology, vol. 17, no. 1, pp. 73-77, 2005.

[6] B. Desir, D. K. Amre, S. E. Lu et al., "Utility of serum antibodies in determining clinical course in pediatric Crohn's disease," Clinical Gastroenterology and Hepatology, vol. 2, no. 2, pp. 139-146, 2004.

[7] U. Müller-Ladner, V. Gross, T. Andus et al., "Distinct patterns of immunoglobulin classes and IgG subclasses of autoantibodies in patients with inflammatory bowel disease," European Journal of Gastroenterology and Hepatology, vol. 8, no. 6, pp. 579-584, 1996.

[8] D. Roggenbuck, G. Hausdorf, L. Martinez-Gamboa et al., "Identification of GP2, the major zymogen granule membrane glycoprotein, as the autoantigen of pancreatic antibodies in Crohn's disease," Gut, vol. 58, no. 12, pp. 1620-1628, 2009.

[9] T. C. Hoops and M. J. Rindler, "Isolation of the cDNA encoding glycoprotein-2 (GP-2), the major zymogen granule membrane protein: homology to uromodulin/Tamm-Horsfall protein," Journal of Biological Chemistry, vol. 266, no. 7, pp. 4257-4263, 1991.

[10] S. I. Fukuoka, S. D. Freedman, and G. A. Scheele, "A single gene encodes membrane-bound and free forms of GP-2, the major glycoprotein in pancreatic secretory (zymogen) granule membranes," Proceedings of the National Academy of Sciences of the United States of America, vol. 88, no. 7, pp. 2898-2902, 1991.

[11] K. Hase, K. Kawano, T. Nochi et al., "Uptake through glycoprotein 2 of $\mathrm{FimH}^{+}$bacteria by $\mathrm{M}$ cells initiates mucosal immune response," Nature, vol. 462, no. 7270, pp. 226-230, 2009.

[12] D. P. Bogdanos, E. I. Rigopoulou, D. S. Smyk, D. Roggenbuck, D. Reinhold, A. Forbes et al., "Diagnostic value, clinical utility and pathogenic significance of reactivity to the molecular targets of Crohn's disease specific-pancreatic autoantibodies," Autoimmunity Reviews, vol. 11, pp. 143-148, 2011.

[13] H. Ohno and K. Hase, "Glycoprotein 2 (GP2) grabbing the fim $\mathrm{H}^{+}$bacteria into $\mathrm{m}$ cells for mucosal immunity," Gut Microbes, vol. 1, no. 6, pp. 407-410, 2010.
[14] J. P. Kraehenbuhl and M. R. Neutra, "Epithelial M cells: differentiation and function," Annual Review of Cell and Developmental Biology, vol. 16, pp. 301-332, 2000.

[15] J. E. Lennard-Jones, "Classification of inflammatory bowel disease," Scandinavian Journal of Gastroenterology, Supplement, vol. 170, pp. 2-6, 1989.

[16] M. S. Silverberg, J. Satsangi, T. Ahmad, I. D. Arnott, C. N. Bernstein, S. R. Brant et al., "Toward an integrated clinical, molecular and serological classification of inflammatory bowel disease: report of a Working Party of the 2005 Montreal World Congress of Gastroenterology," Canadian Journal of Gastroenterology, vol. 19, pp. 5-36, 2005.

[17] D. Roggenbuck, D. Reinhold, T. Wex et al., "Autoantibodies to GP2, the major zymogen granule membrane glycoprotein, are new markers in Crohn's disease," Clinica Chimica Acta, vol. 412, no. 9-10, pp. 718-724, 2011.

[18] D. Roggenbuck, D. Reinhold, T. Wex et al., "Authors' response: antibodies to GP2, the major zymogen granule membrane glycoprotein, are specific for Crohn's disease and may reflect treatment response," Gut, vol. 61, pp. 164-165, 2012.

[19] B. Bonaci-Nikolic, M. Spuran, S. Andrejevic, and M. Nikolic, "Autoantibodies to GP2, the major zymogen granule membrane glycoprotein, in patients with gluten-sensitive enteropathy: a possible serological trap," Clinica Chimica Acta, vol. 413, pp. 822-823, 2012.

[20] P. L. Lakatos, I. Aitorjay, T. Szamosi et al., "Pancreatic autoantibodies are associated with reactivity to microbial antibodies, penetrating disease behavior, perianal disease, and extraintestinal manifestations, but not with NOD2/CARD15 or TLR4 genotype in a Hungarian IBD cohort," Inflammatory Bowel Diseases, vol. 15, no. 3, pp. 365-374, 2009.

[21] K. O. De Beéck, S. Vermeire, P. Rutgeerts, and X. Bossuyt, "Antibodies to GP2, the major zymogen granule membrane glycoprotein, in inflammatory bowel diseases," Gut, vol. 61, pp. 162-164, 2012.

[22] P. Pavlidis, A. Forbes, and D. P. Bogdanos, "Antibodies to glycoprotein 2 (GP2) in patients with inflammatory bowel diseases from UK," Clinica Chimica Acta, vol. 412, no. 11-12, pp. 1163-1164, 2011.

[23] M. Kovacs, P. L. Lakatos, M. Papp, S. Jacobsen, E. Nemes, M. Polgar et al., "Pancreatic autoantibodies and autoantibodies against goblet cells in pediatric patients with inflammatory bowel disease (IBD)," Journal of Pediatric Gastroenterology and Nutrition, vol. 55, no. 4, pp. 429-435, 2012.

[24] M. A. Hölzl, J. Hofer, J. J. Kovarik et al., “The zymogen granule protein 2 (GP2) binds to scavenger receptor expressed on endothelial cells I (SREC-I)," Cellular Immunology, vol. 267, no. 2, pp. 88-93, 2011.

[25] R. L. Owen, "Uptake and transport of intestinal macromolecules and microorganisms by $\mathrm{M}$ cells in Peyer's patches: a personal and historical perspective," Seminars in Immunology, vol. 11, no. 3, pp. 157-163, 1999.

[26] M. R. Neutra, A. Frey, and J. P. Kraehenbuhl, "Epithelial M cells: gateways for mucosal infection and immunization," Cell, vol. 86, no. 3, pp. 345-348, 1996.

[27] K. Hase, S. Ohshima, K. Kawano et al., "Distinct gene expression profiles characterize cellular phenotypes of follicleassociated epithelium and M cells," DNA Research, vol. 12, no. 2, pp. 127-137, 2005.

[28] E. Gullberg and J. D. Söderholm, "Peyer's patches and M cells as potential sites of the inflammatory onset in Crohn's disease," Annals of the New York Academy of Sciences, vol. 1072, pp. 218232, 2006. 
[29] L. Herszenyi and Z. Tulassay, "The role of autoantibodies in inflammatory bowel diseases," Digestive Diseases, vol. 30, pp. 201-207, 2012.

[30] C. Huang and S. Kugathasan, "markers that differentiate early from late IBD,” Digestive Diseases, vol. 30, pp. 380-382, 2012.

[31] A. Kaul, S. Hutfless, L. Liu, T. M. Bayless, M. R. Marahn, and X. $\mathrm{Li}$, "Serum anti-glycan antibody biomarkers for inflammatory bowel diseases diagnosis and progression: a systematic review and metanalysis," Inflammatory Bowel Diseases, vol. 18, no. 10, pp. 1872-1884, 2012.

[32] L. Werner, D. Paclik, C. Fritz, D. Reinhold, D. Roggenbuck, and A. Sturm, "Identification of pancreatic glycoprotein 2 as an endogenous immunomodulator of innate and adaptive immune responses," The Journal of Immunology, vol. 189, no. 6, pp. 2774-2783, 2012. 


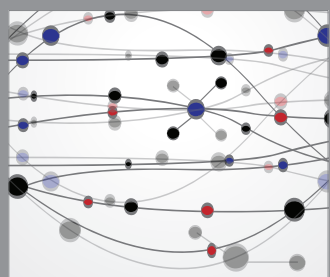

The Scientific World Journal
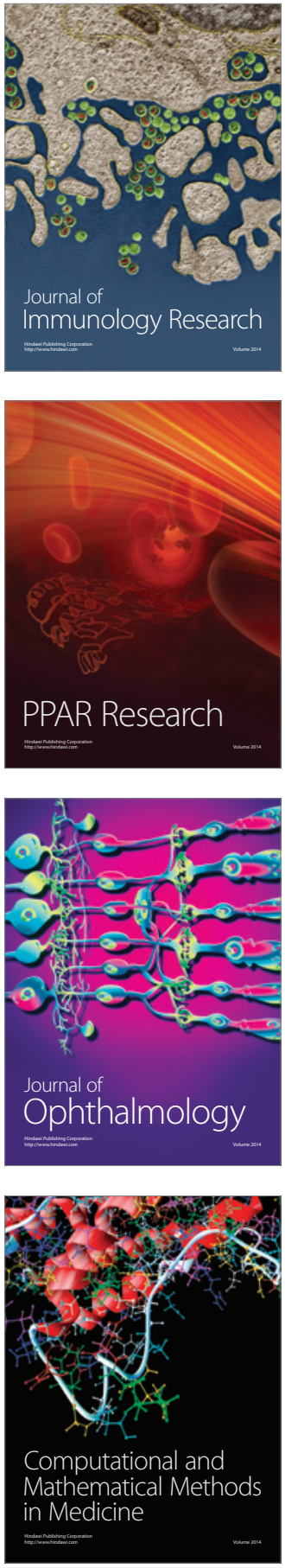

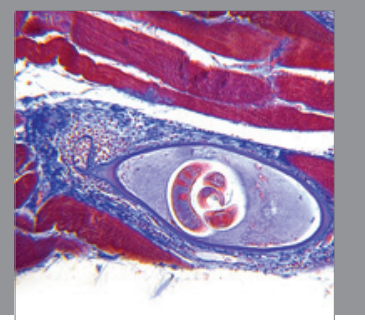

Gastroenterology

Research and Practice
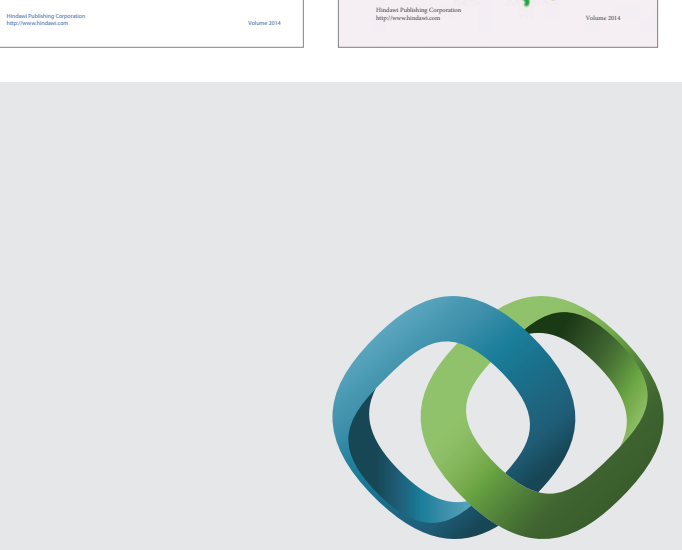

\section{Hindawi}

Submit your manuscripts at

http://www.hindawi.com
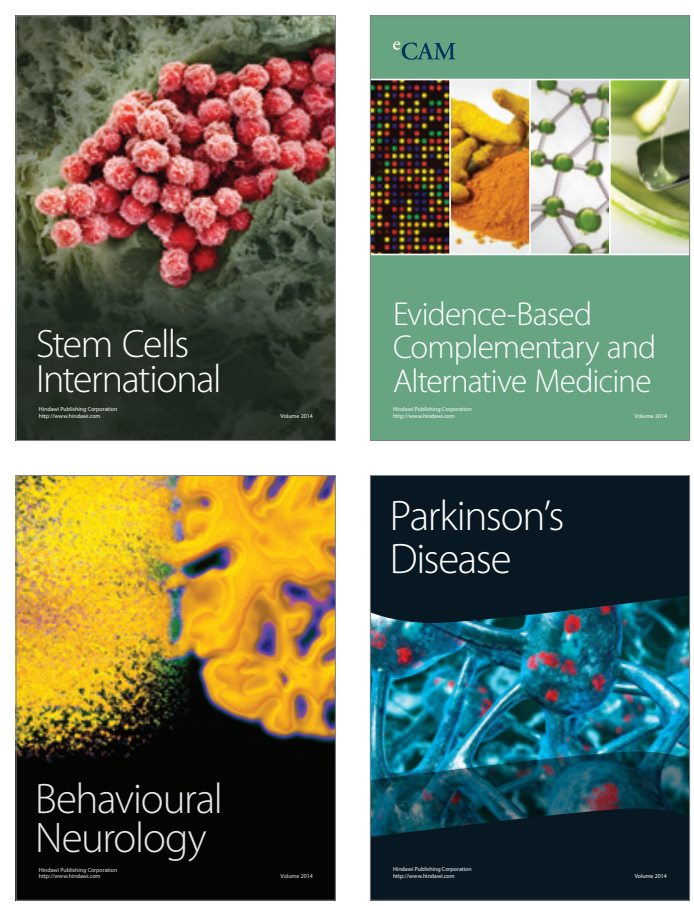

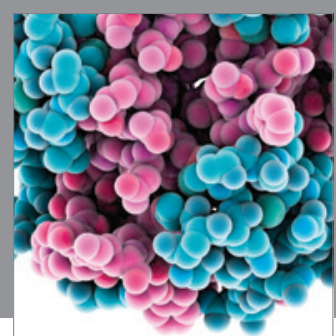

Journal of
Diabetes Research

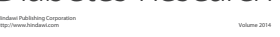

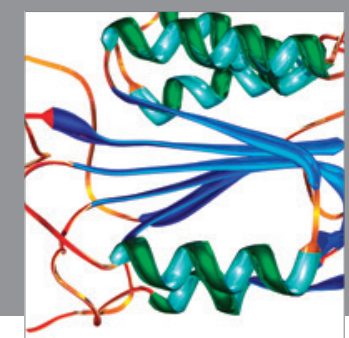

Disease Markers
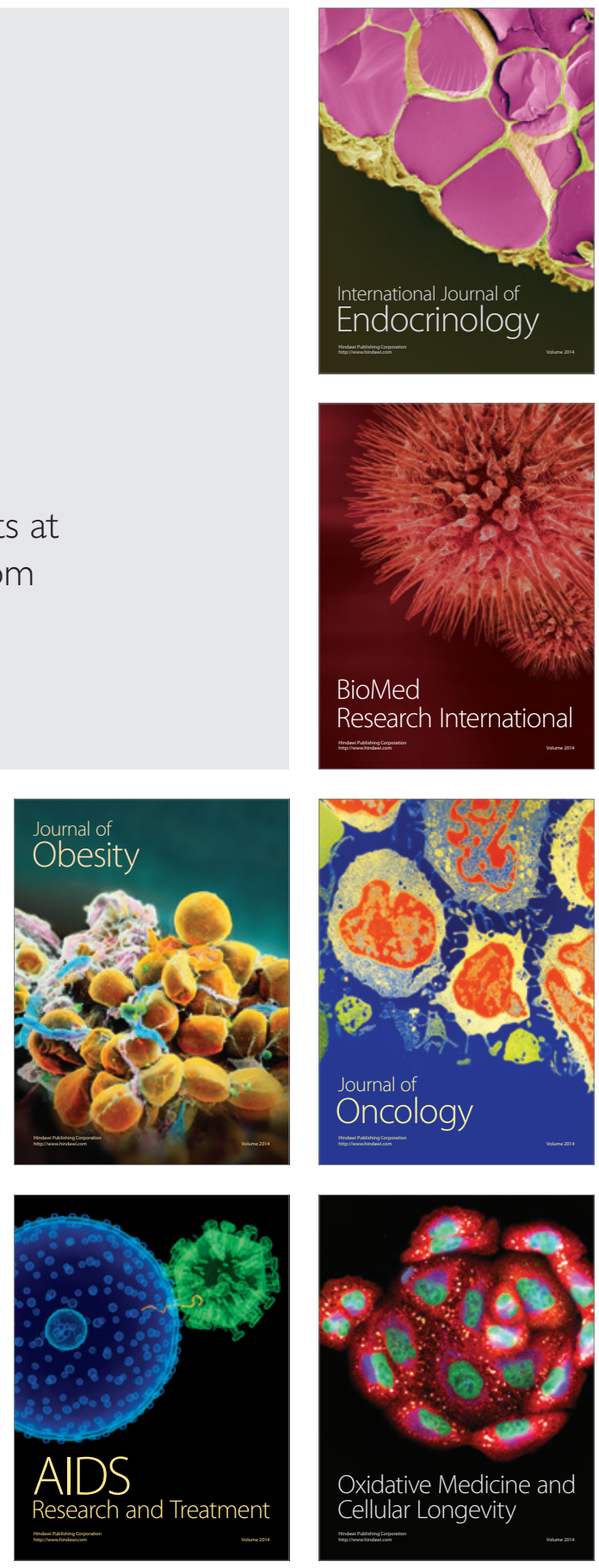